\title{
Epigallocatechin-3-gallate inhibits the proliferation and migration of human ovarian carcinoma cells by modulating p38 kinase and matrix metalloproteinase-2
}

\author{
FENG WANG* ${ }^{*}$ ZHIWEI CHANG* ${ }^{*}$ QINGXIA FAN and LIUXING WANG \\ Department of Oncology, The First Affiliated Hospital of Zhengzhou University, Zhengzhou, Henan 450052, P.R. China
}

Received June 2, 2013; Accepted January 20, 2014

DOI: $10.3892 / \mathrm{mmr} .2014 .1909$

\begin{abstract}
Epigallocatechin-3-gallate (EGCG), a major catechin in green tea, has recently been reported to exhibit anticancer effects on a number of types of cancer cells in vitro; however, the molecular mechanisms of this anticancer effect remain poorly understood. In the current study, the effects of EGCG on the proliferation and migration of the OVCAR-3 human ovarian carcinoma cell line were investigated. Cells were treated with EGCG and their proliferation rates were determined by an MTT assay. In addition, cell migration was detected by transwell assay. The activity of mitogen-activated protein kinases (MAPKs) and the expression of matrix metalloproteinase-2/9 (MMP-2/9) were examined by western blotting. The results showed that EGCG significantly inhibited $(\mathrm{P}<0.05)$ the proliferation of OVCAR-3 cells in a time- and concentration-dependent manner. EGCG $(100 \mu \mathrm{M})$ time-dependently increased $(\mathrm{P}<0.05)$ the activity of $\mathrm{p} 38$, but not extracellular signal-regulated kinases 1/2. SB203580, a specific p38 MAPK inhibitor, completely diminished EGCG-induced phosphorylation of p38 and partially blocked EGCG-inhibited OVCAR-3 cell proliferation. Furthermore, EGCG $(0-100 \mu \mathrm{M})$ dose-dependently inhibited $(\mathrm{P}<0.05)$ OVCAR-3 cell migration. The protein expression levels of MPP-2, but not MMP-9, were dose-dependently decreased following treatment with EGCG $(0-100 \mu \mathrm{M})$ for $48 \mathrm{~h}$. These data indicated that EGCG inhibited OVCAR-3 cell proliferation and migration, potentially mediated via the activation of p38 MAPK and downregulation of the protein expression of MMP2. Thus, the therapeutic potential of EGCG for ovarian cancer requires further investigation.
\end{abstract}

Correspondence to: Dr Liuxing Wang, Department of Oncology, the First Affiliated Hospital of Zhengzhou University, 1 Jianshe Dong Road, Zhengzhou, Henan 450052, P.R. China

E-mail: liuxing_wang@yahoo.com

*Contributed equally

Key words: mitogen-activated protein kinases, proliferation and migration, matrix metalloproteinase-2/9, epigallocatechin-3-gallate, ovarian cancer

\section{Introduction}

Epigallocatechin-3-gallate (EGCG), a major biologically active polyphenol in green tea has long been known to exhibit potential health benefits, including anti-oxidant, anticancer and anti-inflammatory effects (1-3). More recently there have been an increasing number of studies demonstrating the potent anticancer effect of EGCG against various cancer cell lines in vitro, including breast (4), pancreatic (5), colorectal (6) and gastric cancer cell lines (7).

Ovarian cancer is a major cause of mortality among the gynecological malignancies globally. Despite significant improvement in surgical technology and therapy regimens in previous years, the molecular mechanisms underlying the disease progression remain poorly understood $(8,9)$. The development of novel therapeutic agents targeting this potentially fatal gynecological disease is important to improve the prognosis of treatment. However, little is known of the effects of EGCG on human ovarian cancer progression and the associated molecular signaling mechanisms.

It is well demonstrated that a number of cellular, extracellular and cytokine-associated components trigger multiple downstream protein kinase pathways, thus exhibiting a role in the regulation of cell proliferation and migration during cancer development. Among these pathways, the mitogen-activated protein kinases (MAPK) cascades are the most well-studied (10-12). MAPK are proline-directed serine/threonine kinases that have been classified into at least six subfamilies. As the most important member of the MAPKs family, extracellular signal-regulated kinases 1 and 2 (ERK1/2) is required for cell mobility, proliferation and migration $(13,14)$. Another important member of the MAPK family, $\mathrm{p} 38$, is essential in regulating a number of cellular processes, including inflammation, cell differentiation, cell growth and cell death (15-17). However, whether these signaling pathways are involved in EGCG-regulated cell proliferation and migration during ovarian cancer growth remains unknown. In addition, the secretion of matrix metalloproteinases (MMPs) is crucial in cancer cell metastasis and is closely associated with the migration behavior of cancer $(18,19)$. The effects of EGCG on MMP expression in ovarian cancer remain to be investigated.

In the current study, the effect of EGCG on the cell proliferation and migration in OVCAR-3 cells was investigated, as well as the signaling pathways involved in these actions. 


\section{Materials and methods}

Cell line and cell culture. The OVCAR-3 human ovarian adenocarcinoma cell line was obtained from the Cell Bank of Type Culture Collection of Chinese Academy of Sciences (Shanghai, China) and maintained in RPMI-1640 (Gibco-BRL, Life Technologies, Grand Island, NY, USA) supplemented with $10 \%$ fetal bovine serum (FBS, HyClone, Logan, UT, USA), $100 \mathrm{U} / \mathrm{ml}$ penicillin, $100 \mu \mathrm{g} / \mathrm{ml}$ streptomycin and $10 \mathrm{mM}$ L-glutamine. All cells were cultured in a humidified atmosphere of $5 \% \mathrm{CO}_{2}$ at $37^{\circ} \mathrm{C}$. The cells used in this study were at passages $23-26$.

MTT assay. The 3-(4,5-dimethylthiazol-2-yl)-2,5-diphenyl tetrazolium bromide (MTT) viability assay was performed as previously described with slight modifications (17). Briefly, cells were seeded into 96 -well plates with $0.5 \times 10^{4}$ cells/well. Following $16 \mathrm{~h}$ of attachment, different concentrations of EGCG (0-200 $\mu \mathrm{M}$, PeproTech, Rocky Hill, NJ, USA) were applied to the cells in RPMI-1640 culture media and incubated for a further $48 \mathrm{~h}$. The cells were washed with phosphate-buffered saline (PBS) and $200 \mu \mathrm{l}$ MTT $(0.5 \mathrm{mg} / \mathrm{ml})$ was added to each well and further incubated for $4 \mathrm{~h}$. The MTT solution was carefully removed by aspiration and the formazan product was dissolved in $150 \mathrm{ml}$ dimethylsulfoxide. Absorbance was measured at $570 \mathrm{~nm}$ on a microplate reader (BioTek Instruments, Winooski, VT, USA). The same experiments were performed for time-course (2, 4 and 6 days) treatment with $100 \mu \mathrm{M}$ EGCG. To determine the role of the p38 MAPK pathway in cell proliferation, additional cells were subjected to the same assay in the presence of $10 \mu \mathrm{M}$ SB203580 (a specific p38 inhibitor, $1 \mathrm{~h}$ pretreatment; CalBiochem, San Diego, CA, USA). Cell proliferation studies were performed in three independent experiments.

Migration assay. Cell migration was detected using a 24-well transwell chamber with $8.0-\mu \mathrm{m}$ pore polycarbonate filter inserts (Costar, Cambridge, MA, USA). Cells $\left(5 \times 10^{4}\right.$ cells/well) suspended in serum-free RPMI-1640 were overlaid in the upper chamber. In each lower chamber, $800 \mu$ l RPMI-1640 with $10 \%$ FBS in the presence of various concentrations of EGCG $(0-100 \mu \mathrm{M})$ was added. The inserts were incubated at $37^{\circ} \mathrm{C}$ in a humidified atmosphere containing $5 \% \mathrm{CO}_{2}$ for $16 \mathrm{~h}$. Cells that had migrated to the bottom of the inserts were stained with Calcein AM $(0.2 \mathrm{mg} / \mathrm{ml}$; Molecular probes, Eugene, OR, USA) for $30 \mathrm{~min}$, examined and recorded under a microscope (ECLIPSE Ti; Nikon, Tokyo, Japan) mounted with a CCD camera (Nikon). The numbers of migrated cells were counted using the Metamorph image analysis program (Universal Imaging Corporation, West Chester, PA, USA). Cell migration studies were performed in four independent experiments.

Western blotting. Cells were treated with $100 \mu \mathrm{M}$ EGCG for $0-120 \mathrm{~min}$ in RPMI-1640 culture media and in $10 \mu \mathrm{M} \mathrm{SB} 203580$ for $60 \mathrm{~min}$. To determine changes in total and phosphorylated ERK1/2 and p38 protein levels, cells were washed twice with cold PBS, harvested and lysed by sonication (Sonicator 300, Misonix, Inc., Farmingdale, NY, USA) in buffer (4 mM sodium pyrophosphate; $50 \mathrm{mM}$ HEPES, $\mathrm{pH} 7.5 ; 100 \mathrm{mM}$
$\mathrm{NaCl} ; 10 \mathrm{mM}$ EDTA; $10 \mathrm{mM}$ sodium fluoride; $2 \mathrm{mM}$ sodium orthovanadate $\left[\mathrm{Na}_{3} \mathrm{VO}_{4}\right] ; 1 \mathrm{mM}$ PMSF; $1 \%$ Triton X-100; $5 \mathrm{mg} / \mathrm{ml}$ leupeptin and $5 \mathrm{mg} / \mathrm{ml}$ of aprotinin). The protein concentrations in the supernatants of the lysates were determined. Proteins (15-20 $\mu \mathrm{g} / \mathrm{lane})$ were subjected to western blot analysis. Proteins were separated on 10\% SDS-PAGE gels and electroblotted onto Immobilon-P membranes (Millipore, Bedford, MA, USA). Proteins on the membranes were probed with an antibody against total or phospho-specific ERK1/2 (1:2,000 dilution; rabbit polyclonal), total (1:2,000 dilution; rabbit polyclonal) or phospho-specific p38 (1:1,000; dilution; rabbit polyclonal; all Cell Signaling Technology, Inc., Danvers, MA, USA). Changes in total and phosphorylated ERK1/2 and p38 protein levels were quantified. Data on phosphorylated ERK1/2 and p38 were normalized to total ERK1/2 and p38. The same assay was performed on the expression of MMP-2/9 in response to EGCG treatment $(0-100 \mu \mathrm{M})$. Western blotting studies were run in at least three independent experiments.

Statistical analysis. Data were analyzed using one-way analysis of variance (SigmaStat, Jandel Co., San Rafael, CA, USA). When an F-test was significant, data were compared with their respective control by the Bonferroni's multiple comparison test or Student's t-test. $\mathrm{P}<0.05$ was considered to indicate a statistically significant difference.

\section{Results}

EGCG inhibits the growth of human ESCC cells in a timeand dose-dependent manner. EGCG inhibits tumor growth in a number of cancer types (4-7). Based on these studies, the effects of EGCG on the proliferation of ESCC cells were determined. The doses of EGCG used were comparable to those used in previous studies (7). OVCAR-3 cells were treated with EGCG $(0-200 \mu \mathrm{M})$ for $48 \mathrm{~h}$. As shown in Fig. 1A, EGCG dose-dependently $(\mathrm{P}<0.05)$ inhibited the proliferation of OVCAR-3 cells. In addition, the cell proliferation following treatment with EGCG for 2, 4 and 6 days, respectively, was analyzed. As shown in Fig. 1B, EGCG time-dependently $(\mathrm{P}<0.05)$ inhibited OVCAR-3 cell proliferation at a concentration of $100 \mu \mathrm{M}$.

EGCG dose-dependently inhibits OVCAR-3 cell migration. The transwell chamber assay is a commonly used model for analyzing the molecular mechanisms underlying cell migration. In this assay (Fig. 2), EGCG $(0-100 \mu \mathrm{M})$ dose-dependently inhibited $(\mathrm{P}<0.05)$ OVCAR-3 cell migration.

EGCG time-dependently inhibits the phosphorylation of p38, but not ERK1/2. The potential effects of EGCG on the activation of ERK1/2 and p38, which closely associated with carcinoma cell proliferation and migration, were analyzed. As shown in Fig. 3, EGCG time-dependently $(\mathrm{P}<0.05)$ increased the phosphorylation of $\mathrm{p} 38$ in comparison with the time 0 . However, a significant change in the phosphorylation of ERK1/2 was not observed following treatment with $100 \mu \mathrm{M}$ of EGCG.

SB203580 partially blocks EGCG-inhibited OVCAR-3 cell proliferation. To further determine whether the $\mathrm{p} 38$ signaling 
A

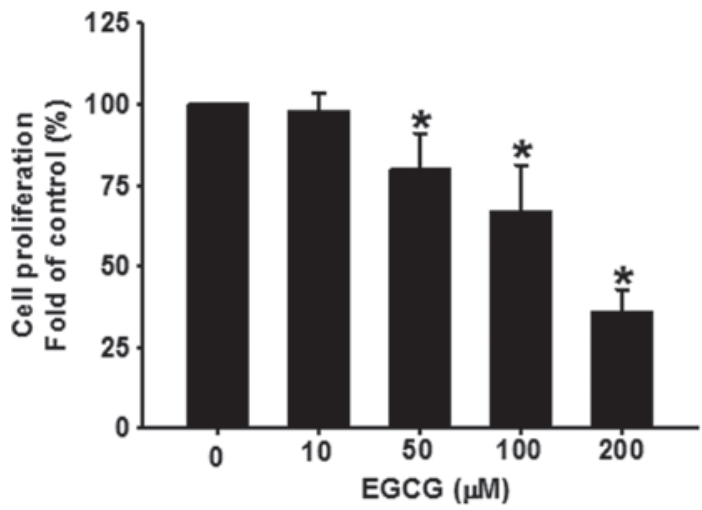

B

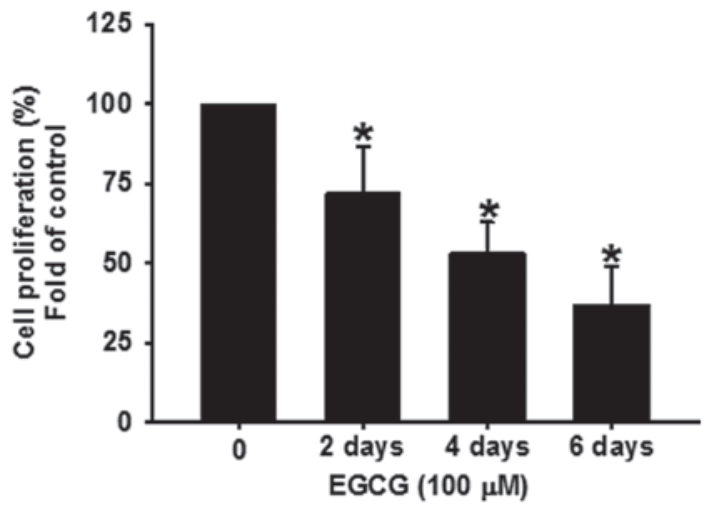

Figure 1. Effects of EGCG on OVCAR-3 cell proliferation using an MTT assay. (A) Cells were exposed to gradually increasing concentrations of EGCG $(0-200 \mu \mathrm{M})$ and incubated for $48 \mathrm{~h}$. (B) Cells were treated with $100 \mu \mathrm{M}$ EGCG for 2, 4 and 6 days, respectively. Cell proliferation was determined by an MTT assay and the graphs showed the results of three independent experiments. The results are presented as a percentage of absorbance relative to that of control cells $(100 \%)$. ${ }^{*} \mathrm{P}<0.05$, vs. control. EGCG, epigallocatechin-3-gallate; MTT, 3-(4,5-dimethylthiazol-2-yl)-2,5-diphenyl tetrazolium bromide.

pathway is involved in the EGCG-inhibited OVCAR-3 cell proliferation, the effects of SB203580 on EGCG regulated cell proliferation were examined. As shown in Fig. 4A and B, SB203580 completely diminished EGCG-induced p38 activation, but partially blocked EGCG inhibited $(\mathrm{P}<0.05)$ OVCAR-3 cell proliferation, suggesting that the p38 signaling pathway may be partially involved in the EGCG-mediated inhibitory effects.

EGCG dose-dependently inhibits the protein expression of $M M P 2$, but not MMP9. Given MMP involvement in the cancer migration process, the effects of EGCG on MMP expression were further investigated by western blotting. As shown in Fig. 5A, following $48 \mathrm{~h}$ treatment, EGCG significantly $(\mathrm{P}<0.05)$ decreased protein expression of MMP-2 at 10,50 and $100 \mu \mathrm{M}$, respectively. However, EGCG did not alter MMP9 and GAPDH protein levels at any time point of the EGCG treatments observed (Fig. 5B).

\section{Discussion}

EGCG is one of the most widely characterized polyphenols in green tea and has been extensively investigated for its chemopreventive effects on tumor formation and development in several types of cancer (4-7). In the present study, significant
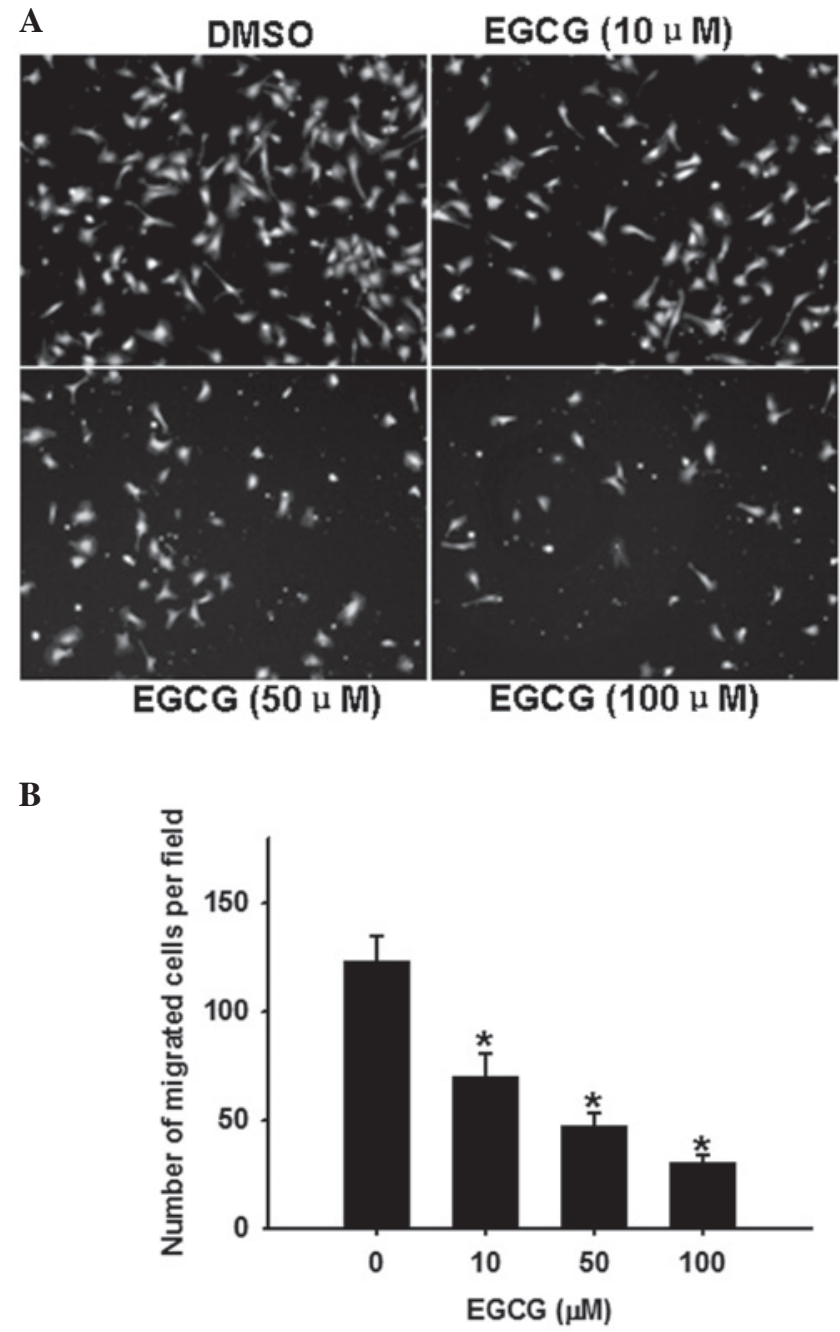

Figure 2. Effects of EGCG on OVCAR-3 cell migration using a transwell assay. Cells suspended in serum-free RPMI-1640 were overlaid in the upper chamber of each transwell. Following incubation with different concentrations of EGCG for $16 \mathrm{~h}$, penetrating cells were stained with Calcein AM and recorded under a microscope mounted with a CCD camera. (A) Images depicted migration of OVCAR-3 cells. (B) Quantified data are expressed as the mean \pm standard error of the mean from four independent experiments. ${ }^{*} \mathrm{P}<0.05$, vs. control. EGCG, epigallocatechin-3-gallate; DMSO, dimethylsulfoxide.

evidence is provided that EGCG is capable of inhibiting proliferation and migration of human ovarian carcinoma cells, partially through the regulation of the activation of p38 kinase and reduction of MMP2 expression, suggesting that EGCG may possess anticancer potential in human ovarian cancer.

An increasing number of studies have indicated that EGCG is capable of inhibiting the growth of various types of cancer cells, including lung (4), colon (6) and gastric (7) cancer cells in culture. This inhibitory effect was also demonstrated in the ovarian cancer cells in the current study. The current data showed that EGCG significantly inhibited ovarian cancer cell growth in a dose- and time-dependent manner. However, little is known of the signaling pathway involved in this inhibitory effect on ovarian cancer growth.

Depending on the cell type, various signaling kinases have been shown to be involved in cell proliferation during tumor development. It is well established that the MAPK family 


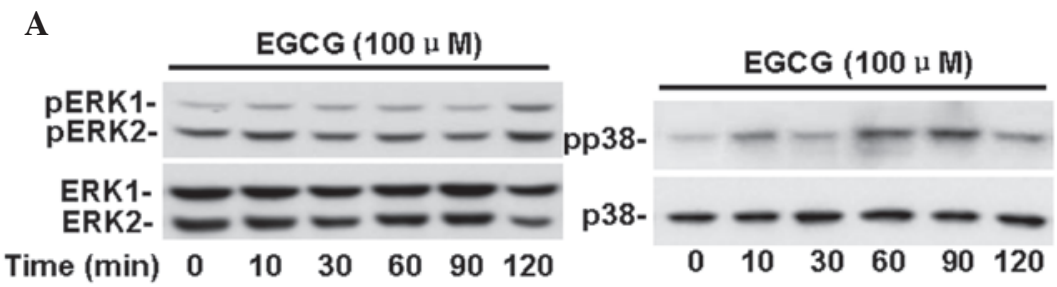

B

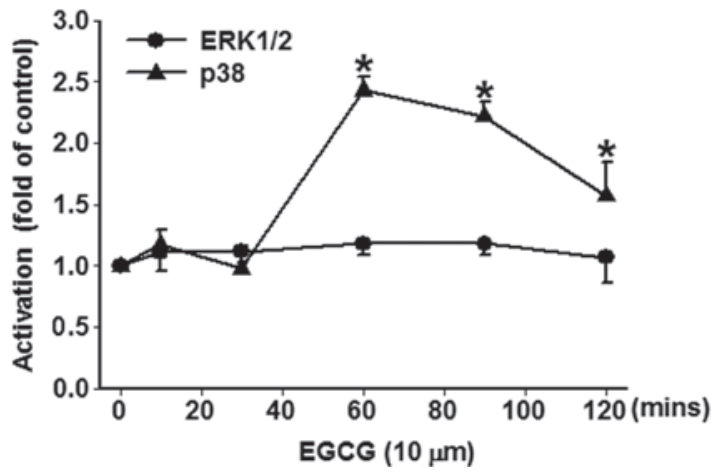

Figure 3. Effects of EGCG on ERK1/2 and p38 phosphorylation in OVCAR-3 cells. Cells were cultured in culture dishes until reaching 60-70\% confluency. Cells were treated with $100 \mu \mathrm{M}$ of EGCG or DMSO for 0-120 min. Proteins were subjected to western blotting and detected using antibodies against phospho-specific and total ERK1/2 or p38. Data normalized to total ERK1/2 or p38 are expressed as means \pm SEM fold of the control from three individual experiments. ${ }^{*} \mathrm{P}<0.05$, vs. control (DMSO treated group). EGCG, epigallocatechin-3-gallate; ERK 1/2, extracellular signal-regulated kinases $1 / 2$.
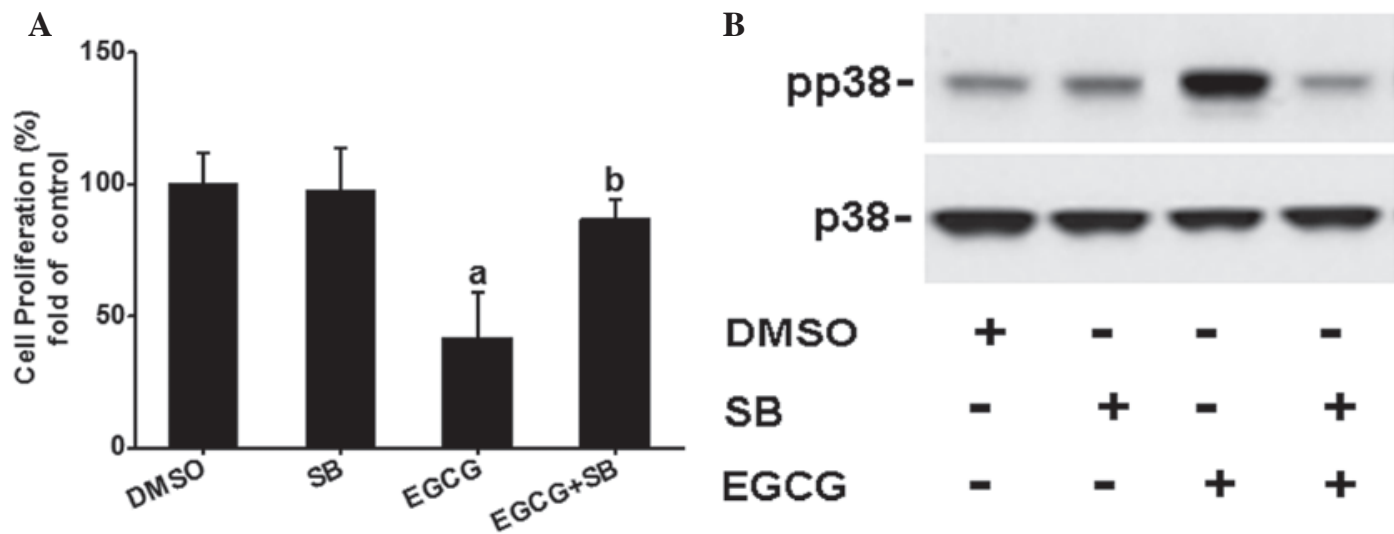

Figure 4. Effects of SB203580 on EGCG-inhibited OVCAR-3 cell proliferation. Cells were treated with $100 \mu \mathrm{M}$ EGCG in the presence or absence of SB for 2 days, respectively. (A) Cell proliferation was determined by an MTT assay and the graphs show the results of four independent experiments. Quantified data are expressed as the mean \pm standard error of the mean from four independent experiments. ${ }^{\mathrm{P}} \mathrm{P}<0.05$ and ${ }^{\mathrm{b}} \mathrm{P}<0.05$, compared with the DMSO control. (B) Phosphorylation of $\mathrm{p} 38$ was detected with western blotting. EGCG, epigallocatechin-3-gallate; SB: SB203580; DMSO, dimethylsulfoxide.

$$
\text { A }
$$

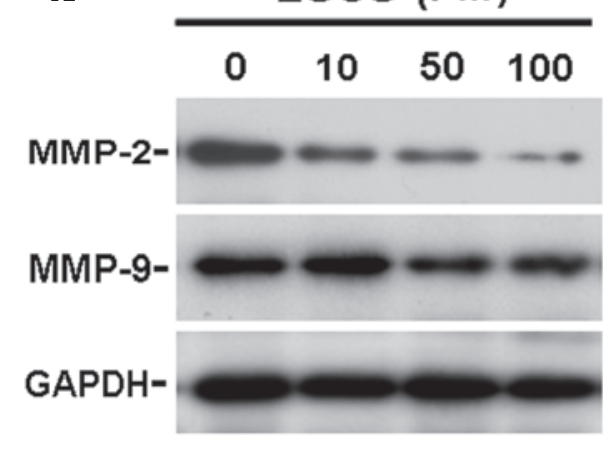

B

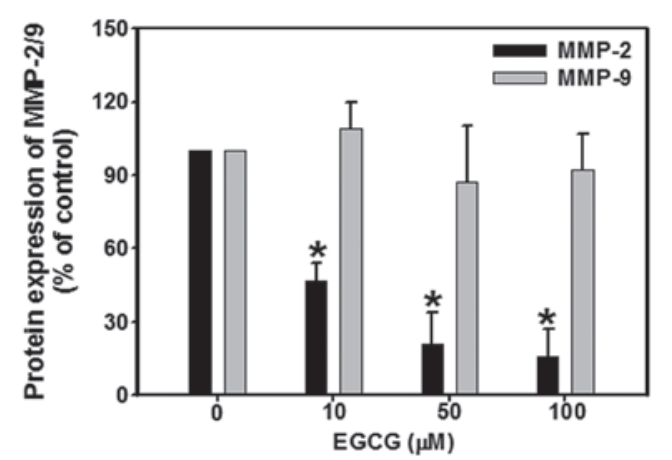

Figure 5. Effects of EGCG on MMP-2/9 protein expression. Cells were cultured in culture dishes until reaching 60-70\% confluency. Cells were treated with EGCG $(0-100 \mu \mathrm{M})$ for $48 \mathrm{~h}$. Proteins were subjected to western blot analysis and detected using antibodies against MMP-2/9 and GAPDH. Data normalized to GAPDH are expressed as the mean \pm standard error of the mean fold of the control from three individual experiments. "P<0.05, vs. control. EGCG, epigallocatechin-3-gallate. MMP-2/9, matrix metalloproteinase-2/9. 
are actively involved in the regulation of cellular functions, particularly of cell proliferation $(20,21)$. A previous study by Shankar et al (5) showed that EGCG significantly reduced ERK activity and enhanced p38 and JNK activities in a human pancreatic tumor xenograft model. In accordance with this report, the current study showed that EGCG significantly activated p38 in a time-dependent manner. SB203580, the specific inhibitor of p38, completely inhibited the EGCG-induced increased phosphorylation of p38. However, significant changes in the activity of ERK1/2 in response to EGCG were not observed in the present data, which may be attributable to the different cell types.

Cancer metastasis is a highly coordinated multistep process involving cell invasion, cell-cell and cell-matrix adhesion and remodeling of the extracellular matrix $(22,23)$. The current study indicated that EGCG dose-dependently inhibits OVCAR-3 cell migration. However, the molecular mechanisms underlying this effect remain unknown. It is well observed that a number of proteinases are involved in the degradation of the ECM by cancer cells, including MMPs, serine proteinase, and in particular, members of the uPA-plasmin system (24). The secretion of MMPs is crucial in cancer cell metastasis and is involved in cancer cell migration and adhesion. Among human MMPs, MMP-2/9 are abundantly expressed in various malignant tumors, including ovarian cancer (25-27). The present data demonstrated that EGCG significantly decreased the expression of MMP-2, but not MMP-9, which is also supported by previous studies where EGCG reduced MMP-2 expression in human lung and breast cancer cells $(28,29)$.

In conclusion, this preliminary investigation has shown that the anticancer effect of EGCG in an ovarian cancer cell model may be mediated via the activation of p38 MAPK signaling pathway as well as the decreased expression of MMP-2. These findings reveal EGCG as a potential novel therapeutic anticancer therapy.

\section{Acknowledgements}

This study was supported by a grant from the Youth Innovation Foundation of the First Affiliated Hospital of Zhengzhou University.

\section{References}

1. Katiyar SK and Elmets CA: Green tea polyphenolic antioxidants and skin photoprotection (Review). Int J Oncol 18: 1307-1313, 2001.

2. Singh BN, Shankar S and Srivastava RK: Green tea catechin, epigallocatechin-3-gallate (EGCG): mechanisms, perspectives and clinical applications. Biochem Pharmacol 82: 1807-1821, 2011.

3. Mukhtar H and Ahmad N: Green tea in chemoprevention of cancer. Toxicol Sci 52 (2 Suppl): S111-S117, 1999.

4. Hsu YC and Liou YM: The anti-cancer effects of (-)-epigallocatechin-3-gallate on the signaling pathways associated with membrane receptors in MCF-7 cells. J Cell Physiol 226: 2721-2730, 2011.

5. Shankar S, Ganapathy S, Hingorani SR and Srivastava RK: EGCG inhibits growth, invasion, angiogenesis and metastasis of pancreatic cancer. Front Biosci 13: 440-452, 2008.

6. Sukhthankar M, Alberti S and Baek SJ: (-)-Epigallocatechin-3-gallate (EGCG) post-transcriptionally and post-translationally suppresses the cell proliferative protein TROP2 in human colorectal cancer cells. Anticancer Res 30: 2497-2503, 2010.
7. Onoda C, Kuribayashi K, Nirasawa S, Tsuji N, Tanaka M, Kobayashi D and Watanabe N: (-)-Epigallocatechin-3-gallate induces apoptosis in gastric cancer cell lines by down-regulating survivin expression. Int J Oncol 38: 1403-1408, 2011.

8. Cho KR and Shih IeM: Ovarian cancer. Annu Rev Pathol 4: 287-313, 2009.

9. Urban $\mathrm{N}$ and Drescher C: Potential and limitations in early diagnosis of ovarian cancer. Adv Exp Med Biol 622: 3-14, 2008.

10. Mizutani K,ItoH,Iwamoto I, et al: Essential roles of ERK-mediated phosphorylation of vinexin in cell spreading, migration and anchorage-independent growth. Oncogene 26: 7122-7131, 2007.

11. Ray RM, Vaidya RJ and Johnson LR: MEK/ERK regulates adherens junctions and migration through Rac1. Cell Motil Cytoskeleton 64: 143-156, 2007.

12. Kim EK and Choi EJ: Pathological roles of MAPK signaling pathways in human diseases. Biochim Biophys Acta 1802: 396-405, 2010.

13. Kang YH, Yang IJ and Shin HM: Herbal formula HMC05 prevents human aortic smooth muscle cell migration and proliferation by inhibiting the ERK1/2 MAPK signaling cascade. J Nat Med 66: 177-184, 2012.

14. Gayer CP, Craig DH, Flanigan TL, Reed TD, Cress DE and Basson MD: ERK regulates strain-induced migration and proliferation from different subcellular locations. J Cell Biochem 109: 711-725, 2010.

15. Tangkijvanich $P$, Santiskulvong $C$, Melton AC, Rozengurt E and Yee HF Jr: p38 MAP kinase mediates platelet-derived growth factor-stimulated migration of hepatic myofibroblasts. J Cell Physiol 191: 351-361, 2002.

16. Li T, Feng Z, Jia S, Wang W, Du Z, Chen N and Chen Z: Daintain/AIF-1 promotes breast cancer cell migration by up-regulated TNF- $\alpha$ via activate p38 MAPK signaling pathway. Breast Cancer Res Treat 131: 891-898, 2012.

17. Xue A, Xue M, Jackson C and Smith RC: Suppression of urokinase plasminogen activator receptor inhibits proliferation and migration of pancreatic adenocarcinoma cells via regulation of ERK/p38 signaling. Int J Biochem Cell Biol 41: 1731-1738, 2009.

18. Choi BD, Jeong SJ, Wang G, Park JJ, Lim DS, Kim BH, Cho YI, Kim CS and Jeong MJ: Secretory leukocyte protease inhibitor is associated with MMP-2 and MMP-9 to promote migration and invasion in SNU638 gastric cancer cells. Int J Mol Med 28: 527-534, 2011.

19. Malemud CJ: Matrix metalloproteinases (MMPs) in health and disease: an overview. Front Biosci 11: 1696-1701, 2006.

20. HilgerRA,Scheulen MEand StrumbergD:The Ras-Raf-MEK-ERK pathway in the treatment of cancer. Onkologie 25: 511-518, 2002.

21. Geest CR and Coffer PJ: MAPK signaling pathways in the regulation of hematopoiesis. J Leukoc Biol 86: 237-250, 2009.

22. Brooks SA, Lomax-Browne HJ, Carter TM, et al: Molecular interactions in cancer cell metastasis. Acta Histochem 112: 3-25, 2010.

23. Bourboulia D and Stetler-Stevenson WG: Matrix metalloproteinases (MMPs) and tissue inhibitors of metalloproteinases (TIMPs): positive and negative regulators in tumor cell adhesion. Semin Cancer Biol 20: 161-168, 2010.

24. Roy R, Yang J and Moses MA: Matrix metalloproteinases as novel biomarkers and potential therapeutic targets in human cancer. J Clin Oncol 27: 5287-5297, 2009.

25. Hwang ES and Park KK: Magnolol suppresses metastasis via inhibition of invasion, migration, and matrix metalloproteinase-2/-9 activities in PC-3 human prostate carcinoma cells. Biosci Biotechnol Biochem 74: 961-967, 2010.

26. Sun LC, Luo J, Mackey LV, Fuselier JA and Coy DH: A conjugate of camptothecin and a somatostatin analog against prostate cancer cell invasion via a possible signaling pathway involving PI3K/Akt, alphaVbeta3/alphaVbeta5 and MMP-2/-9. Cancer Lett 246: 157-166, 2007.

27. Schmalfeldt B, Prechtel D, Härting K, Späthe K, Rutke S, Konik E, Fridman R, Berger U, Schmitt M, Kuhn W and Lengyel E: Increased expression of matrix metalloproteinases (MMP)-2, MMP-9, and the urokinase-type plasminogen activator is associated with progression from benign to advanced ovarian cancer. Clin Cancer Res 7: 2396-2404, 2001.

28. Deng YT and Lin JK: EGCG inhibits the invasion of highly invasive CL1-5 lung cancer cells through suppressing MMP-2 expression via JNK signaling and induces G2/M arrest. J Agric Food Chem 59: 13318-13327, 2011.

29. Sen T, Moulik S, Dutta A, Choudhury PR, Banerji A, Das S, Roy M and Chatterjee A:Multifunctionaleffect of epigallocatechin-3-gallate (EGCG) in downregulation of gelatinase-A (MMP-2) in human breast cancer cell line MCF-7. Life Sci 84: 194-204, 2009. 